\title{
A Comparison of Data Association Techniques for Target Tracking in Clutter
}

\author{
Ahmed Gad \\ Department of Electrical and Computer \\ Engineering, \\ Royal Military College of Canada, \\ Kingston, Ontario, Canada, K7K 7B4 \\ ahmed.gad@rmc.ca
}

\author{
F. Majdi \\ Department of Electrical and Computer \\ Engineering, \\ Royal Military College of Canada, \\ Kingston, Ontario, Canada, K7K 7B4 \\ majdi-f@rmc.ca
}

\author{
M. Farooq \\ Department of Electrical and Computer \\ Engineering, \\ Roy al Military College of Canada, \\ Kingston, Ontario, Canada, K7K 7B4 \\ farooq@rmc.ca
}

\begin{abstract}
In tracking a single target in clutter, many algorithms have been developed ranging in complexity from nearest neighbor (NN) and probabilistic data association (PDA) to the optimal Bayesian filter. In multiple-target tracking, a number of the techniques have been exercised such as the JPDA and the multiple hypothesis (MHT) schemes. Sub-optimal algorithms, such as the PDA filter, have been used widely since the optimal algorithms have an exponentially increasing computational complexity since all the possible sequences of target-to-measurement associations must be considered. In this paper, the Viterbi algorithm (VA) is used to develop a parallel search data association algorithm, called the Viterbi Data Association (VDA) technique. This algorithm includes the gating, automatic track initiation and termination modules. Simulations have been carried out to verify the performance and the robustness of the proposed algorithm. Moreover, the $V D A$ algorithm is compared with the fuzzy data association (FDA) algorithm when tracking a target in a cluttered, low signal-to-noise ratio (SNR) environment.
\end{abstract}

Keywords: Target Tracking, Data Association, Viterbi Algorithm, Dynamic Programming, Fuzzy logic, Probabilistic Data Association, Clutter Environment.

\section{Introduction}

The VA is a recursive algorithm, which provides a solution to the discrete linear optimization problem. The VA is based on a trellis diagram in which from a given a sequence of symbols, the most-likely state transition sequence in a state diagram is determined [1,2]. The basis of the algorithm is built upon the Markov Process.

In short, the main idea of the VA is to determine the shortest (optimum) path across the trellis. Due to its ease of implementation and computational simplicity, the VA has been applied to many different areas in decoding such as, text recognition, as well as estimation and detection problems in digital communications [3]. Recently it has also found applications in target detection and tracking $[4,5]$.
The PDA algorithm assumes that only one target is present and a track exists for this target with at least one validated measurement. All other measurements are assumed to be from clutter. This algorithm is based upon the Bayesian approach. [10,11].

Conventional tracking techniques use the "all-or-none" criterion to the tracking problem. However, in real world scenarios, the regions around the target detection threshold and the data association gate boundary are actually fuzzy. Thus there is no distinct boundary, separating target from non-target measurements. Therefore, in practice, regardless of the level of sophistication of the tracker, its performance will not match that of an experienced human operator. A more recent technique which is gaining popularity is fuzzy logic, which can be used to simulate the fuzzy decisions.

\section{The Viterbi Concept}

The basis of the VA is built upon the Markov process (MP), [6, 7]. The MP uses state diagrams where the "nodes" (circles) represent the state and the "arcs" are represented by the transition paths. As time progresses, the process will trace some path from state to state through the state diagram. If the states and their transitions are repeated in time for $\mathrm{k}=1, . ., \mathrm{T}$, we obtain what is called a trellis. A trellis is a directed graph consisting of a set of nodes $(\mathrm{N})$ and directed arcs $(\mathrm{A})$ or paths $(\mathrm{P})$ as shown in figure 1 .

At each node in each transition, the length of the paths coming into the node is summed. The path with the shortest length is considered the survivor of that round and its length will be assigned to that node. Over all, there will be $N$ survivors, one for each node. Therefore, the recursion will proceed from first to last state, without ever exceeding $N$. This algorithm can be viewed as a simple version of forward dynamic programming. However, once the last node is reached, the algorithm backtracks, summing all the smallest nodes from each previous state to obtain the optimal path. 


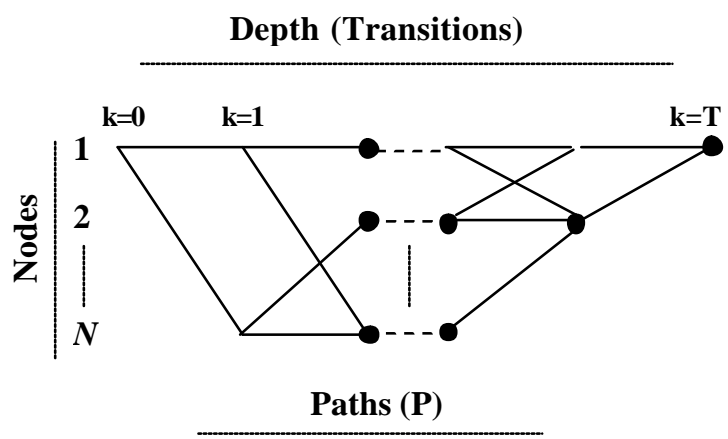

Fig. 1 Trellis Diagram and the application of VA

\section{The Application of Viterbi to Target Tracking}

The study of target detection and tracking in a cluttered environment has been well studied [8-12]. In multiple object detection, there are two main concepts to consider, namely: data association and state estimation. In other words, not only we need to determine the correct object, we need to estimate its state with the given measurements.

A number of statistical based solutions to the data association problem have been explored such as the PDA, MHT, and the 0-1 integer programming algorithm. The PDA is based on a many-measurement-to-one-track type assignment and ,thus, will have a poor performance in a high clutter environment. The latter two algorithms are based on a one-to-one track type assignment, thus having a much better performance in a high clutter environment. However, the main difficulty with their implementation is that they are computationally inefficient and require a high memory storage space.

\subsection{Target Tracking Model}

Based on the work of Quach and Farooq [4], the state dynamic equations and the observation equations of a single target are defined as follows:

$$
\begin{aligned}
& x_{k}=\phi_{k, k-1} x_{k-1}+w_{k-1} \\
& Z_{k}=H_{k} x_{k-1}+v_{k-1}
\end{aligned}
$$

where $\mathrm{x}_{\mathrm{k}}$ is the target state vector of dimension $\mathrm{p}$ at time $\mathrm{k}$; $\phi_{k, k-1}$ is a $p \times p$ transition matrix; $\mathrm{w}_{\mathrm{k}}$ is a zero mean white Gaussian plant noise with $E\left\{\left(w_{i}\right)\left(w_{i}\right)^{T}\right\}=Q_{i} \delta_{i j} ; \mathrm{z}_{\mathrm{k}}$ is the observation vector of dimension $\mathrm{q}$ at time $\mathrm{k} ; \mathrm{H}_{\mathrm{k}}$ is a $q \times p$ observation matrix; and $\mathrm{v}_{\mathrm{k}}$ is also a zero mean white Gaussian measurement noise with $E\left\{\left(v_{i}\right)\left(v_{i}\right)^{T}\right\}=R_{i} \delta_{i j}$ (the noise vectors $\mathrm{w}_{\mathrm{k}}$ and $\mathrm{v}_{\mathrm{k}}$ are uncorrelated, i.e., $\left.E\left\{\left(w_{i}\right)\left(v_{i}\right)^{T}\right\}=0\right)$. Similar to that in [4], we assumed the signal state to be: $x_{k}=[\beta, \dot{\beta}, f, \dot{f}]^{T}$ (the vector parameters denote the source bearing, source bearing rate, source frequency, source frequency rate respectively).

The measurement is modeled as:

$$
z_{k j}=\left\{\begin{array}{cl}
H_{k} x_{k}+v_{k} & \text { if from target } \\
\omega_{k} & \text { otherwise }
\end{array}\right.
$$

where $\omega_{\mathrm{k}}$ is a clutter, uniformly distributed over the surveillance volume.

By convention, the set of measurements at scan $\mathrm{k}$ is denoted by $Z_{k}$ and the collection of all the cumulative measurements is represented as $Z^{T}=\left\{Z_{i}\right\}_{i=1}^{T}$, where $\mathrm{T}$ is the number of stages.

\subsection{The Viterbi Algorithm}

The Viterbi algorithm is a recursive algorithm that provides a solution to a discrete linear optimization problem by determining an optimal state sequence on a trellis diagram. A trellis diagram is a type of directed graph that consists of a set of nodes $(N)$ and a set of directed $\operatorname{arcs}(A)$. The nodes $\left(n_{i}^{k}\right)$ are partitioned into ordered sets with the k-th set being denoted as $\mathrm{N}^{\mathrm{k}}$, where $\mathrm{k}$ represents the stages in the trellis $(\mathrm{k}=1,2, \ldots, \mathrm{T})$. The number of nodes in each stage is m which may be different at each stage. The set of $\operatorname{arcs} A$ is a collection of ordered pairs $\left\{n_{i}^{1}, n_{j}^{k}\right\}$, which implies some logical relationship between the two nodes. The set of directed arcs $A$ consists of only those arcs represented by $\left\{n_{i}^{k-1}, n_{j}^{k}\right\}$, where $\mathrm{k}=2, \ldots, \mathrm{T}$.

A path $\mathrm{P}$ is a collection of directed arcs that connects an element at stage 1 to an element at stage T. Each directed arcs is associated with a metric or a distance label $a_{i j}^{k}$. A path metric is defined as the sum of the metrics of all the arcs contained in the path $\mathrm{P}$ as

$$
d(P)=\sum_{k=2}^{T} a_{i j}^{k} ; \quad\left\{n_{i}^{k-1}, n_{i}^{k}\right\} \in P
$$

The Viterbi Algorithm is based on traversing a trellis from the first state to the last via arcs connecting the nodes. Thus, once the last node is reached, the algorithm backtracks all the smallest nodes from each previous state to obtain the optimal path. A summary of the Viterbi Algorithm is given below; however, in order to enhance the understanding of the algorithm the notations used will be defined first. 
Notations used in the VA:

$d_{i}^{k}$ is the metric of node $n_{i}^{k} ; \psi(k, i)$ is the predecessor function of node $n_{i}^{k} ; \mathrm{i}, \mathrm{j}$ are indices of elements in $\mathrm{N}^{k}$; and $\mathrm{d}^{\mathrm{T}^{*}}$ is the metric of the shortest path in the trellis diagram.

To solve the data association problem, let each node represent an observation. The collection of measurements at the $\mathrm{k}$-th scan $\mathrm{Z}_{\mathrm{k}}$ corresponds to the set of nodes at k-th stage of each trellis. Thus, we can set-up a trellis diagram such that there is a one-to-one correspondence between the set of nodes $N$ in the trellis and the set of measurements in the cumulative set $Z^{T}$. Furthermore, we define the arcs on the trellis as representations of the joint likelihood probabilities $p\left(z_{(k-1) i}, z_{k j} / x_{k-1}, x_{k}\right)$, where $\mathrm{x}_{\mathrm{k}-1}$ and $\mathrm{x}_{\mathrm{k}}$ are the true target states at time (k-1) and time k, respectively.

The distance label of a node $n_{j}^{k}$ is a joint likelihood probability of the sequence of nodes terminating at this node i.e., $p\left(z_{k j}, Z[\psi(k-1, i)] / x_{k}, X^{k-1}\right)$, where $\Psi$ is the set of indices of the nodes, and $Z[\Psi(k-1, i)]$ is the sequence of nodes which belong to a path that terminates at the node $n_{i}^{k-1}$. For simplicity, define the distance metric at node $n_{i}^{k}$ to be a negative log likelihood function, that is $-\ln \left\{p\left(z_{k j}, Z[\psi(k-1, i)] / x_{k}, X^{k-1}\right)\right\}$, with $\mathrm{X}^{\mathrm{k}-1}$ being the set of true past target states. Thus, within the context of the VA, the node metric $d_{i}^{k}$ is simply the log likelihood function of the best assignment sequence that includes the measurement associated with the node. By applying the Viterbi algorithm to the trellis diagram, we obtain the sequence of measurements that maximizes the overall likelihood function, thus solving the data association problem.

\subsection{Viterbi Algorithm used in the Tracking Simulations}

The VA-based tracking algorithm simu lated in this paper can be summarized as follows (see figure 2):

1) Initialization step: assign a value of zero to the label at each node in stage 1 :

$$
\begin{array}{ll}
\text { Score }(1, \mathrm{i})=0, & 1 \leq i \leq m_{1} \\
\Psi(\mathrm{k}, \mathrm{i})=0, & 1 \leq i \leq m_{1}
\end{array}
$$

2) Repeat the following procedure for each stage $k$, where $\mathrm{k}=2, \ldots, \mathrm{T}$

- For each node $\mathrm{i}=0, \ldots, \mathrm{m}_{\mathrm{k}}$ (at stage $\mathrm{k}-1$ ), calculate the predicted position conditioned on the measurement sequence terminating at the node $n_{i}^{k-1}$.

$$
\begin{aligned}
& \hat{x}_{k / k-1}^{i}=\Phi \hat{x}_{k-1 / k-1}^{i} \\
& P_{k / k-1}^{i}=\Phi P_{k-1 / k-1}^{i} \Phi^{T}+Q_{k-1} \\
& S_{k / k-1}^{i}=H S_{k-1 / k-1}^{i} H^{T}+R_{k-1}
\end{aligned}
$$

- For each node $\mathrm{j}=0, \ldots, \mathrm{m}_{\mathrm{k}}$ (at stage $\mathrm{k}$ ), calculate the distance label $a_{i j}^{k}$ of the arc joining nodes $n_{i}^{k-1}$ and $n_{i}^{k}$ as given in reference [4].

$a_{i j}^{k}=\left\{\begin{array}{c}-\ln \left\{\Lambda\left(z_{k j} / x_{k}, Z\left[\Psi^{k-1}(i)\right]\right)\right\}, \quad j \neq 0, i=1, \ldots, m_{k-1} \\ 0, \quad j=0, i=1, \ldots, m_{k-1}\end{array}\right.$

where

$$
\begin{aligned}
& -\ln \{\Lambda(.)\}=\left[0.5\left(\tilde{z}_{k}^{i j}\right)^{T}\left(S_{k}^{i}\right)^{-1}\left(\tilde{z}_{k}^{i j}\right)\right]+a_{1}(\Delta \dot{\hat{\beta}})^{2}+ \\
& a_{2}(\Delta \dot{\hat{f}})^{2}-\ln \left\{a_{3} \frac{P_{D} V_{s}}{\left(1-P_{D}\right)}\right\}+\ln \left\{2 \pi\left|S_{k}^{i}\right|^{0.5}\right\}
\end{aligned}
$$

where $(\Delta \dot{\hat{\beta}})^{2}=\dot{\hat{\beta}}_{k}-\dot{\hat{\beta}}_{k-1}$ and $(\Delta \dot{\hat{f}})^{2}=\dot{\hat{f}}_{k}-\dot{\hat{f}}_{k-1}$ are the estimated deviations in bearing rate and frequency rate at $\mathrm{k}$, respectively. $\mathrm{a}_{1}$ and $\mathrm{a}_{2}$ are constants determined through experimentation. The main idea here is to include a penalty term for deviations in the velocity components. Due to the inclusion of the penalty terms for the deviations in the velocity components, the missed detection threshold needs also be artificially increased by $\mathrm{a}_{3}$ so that the modification will work properly. In our simulation, the $a_{3}$ is linearly increased with the number of missed detection scans; thus, during a track loss scenario (indicated by a large number of missed detection scans), equation (11) will force the VA to seek sequence of measurements with minimum changes in the velocity components.

- Assign node $n_{i}^{k}$ with the smallest label as

$$
\begin{aligned}
& \text { follows: } i^{*}=\arg \left\{\min _{0 \leq i \leq m_{1}-1}\left\{d_{i}^{k-1}+a_{i j}^{k}\right\}\right\} \\
& \operatorname{Score}(k, j)=\left(d_{i}^{k-1}+a_{i j}^{k}\right) \\
& \psi(k, j)=i^{*}
\end{aligned}
$$

- Update the target state at each node in stage $\mathrm{k}$

$$
\begin{aligned}
& K_{k}^{j}=P_{k / k-1}^{i} H^{T}\left[H P_{k / k-1}^{i} H^{T}+R_{k}\right]^{-1} \\
& P_{k / k-1}^{i^{i}}=\left[I-G_{k}^{i} H\right] P_{k / k-1}^{i} \\
& \hat{x}_{k / k}^{j} \stackrel{\Delta}{=} x_{k / k-1}^{i}+K_{k}^{j}\left[z_{k j}-\hat{x}_{k / k-1}^{j}\right]
\end{aligned}
$$

3) Final Selection: Determine the node with the minimum score in the final stage. The current state of the target is the state associated with the minimal node. $i^{*}=\arg \left\{\min _{0 \leq i \leq m_{-}-1}\{\operatorname{score}(k, i)\}\right\}$

$$
\hat{x}_{T}=\hat{x}_{T / T}^{i_{i}^{*}}
$$

4) Back track: recover the measurement sequence that terminates with the minimum node score in the final stage using

$$
i_{k-1}^{*}=\psi\left(k, i_{k}^{*}\right) \quad k=T, T-1, . .2
$$




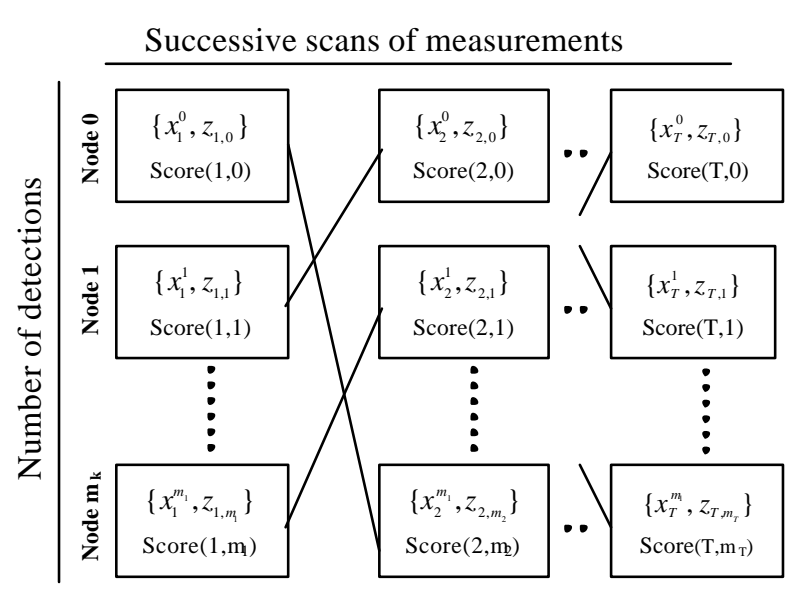

Fig. 2 Trellis Diagram for Target Tracking

\section{PDA Application to Target Tracking}

The PDA algorithm is based on the Bayesian approach, which calculates the probability that each measurement in the validation region originated from the target and the probability that none of the validated measurements are from the target. The target states are updated using the calculated probabilities and the validated measurements $[10,11]$.

The Kalman filter estimates the target states from the observations that are corrupted by random noise. The target probability of detection depends mainly on the signal strength and the sensor accuracy. The PDAF can overcome the uncertainty of the measurements. This improvement is achieved by gating the measurements around the predicted target position and if the target is detected, then the new measurement should fall in this data association gate with high probability and any measurement falling outside the gate can be rejected.

In the PDA algorithm, a composite estimate is obtained as a weighted-sum of all the estimates generated from the measurements inside the gate. The weight assigned to each estimate is proportional to the probability that the estimate originates from the target.

The PDAF algorithm from references [10-11] is briefly discussed below. For $m$ measurements falling in the validation gate at $t_{k}$, the probability that the $j^{\text {th }}$ validated measurement $z_{j}(k)$ is from the target, denoted $\beta_{\mathrm{j}}$, is

$$
\beta_{j}=\frac{e_{j}}{b+\sum_{\ell=1}^{m_{k}} e_{\ell}} \quad\left(\mathrm{j}=1, \ldots, \mathrm{m}_{\mathrm{k}}\right),
$$

while the probability that none of the measurements is from the target, denoted $\beta 0$, is

$$
\beta_{0}=\frac{b}{b+\sum_{\ell=1}^{m_{k}} e_{\ell}} .
$$

The term $e_{j}$ is given by

$$
e_{j}=\exp \left\{-\frac{1}{2} v_{j k}^{T} S_{k}^{-1} v_{j k}\right\},
$$

where $v_{j k}$ is the residual for the $\mathrm{j}^{\text {th }}$ validated measurement and $S_{k}$ is the residual covariance for the measurements. The term $b$ is given by

$$
b=\lambda \sqrt{\operatorname{det}\left[2 \pi S_{k}\right]} \frac{1-P_{D} P_{G}}{P_{D}},
$$

where $\lambda$ is the spatial density of the clutter, $P_{D}$ is the detection probability, and $\mathrm{P}_{\mathrm{G}}$ is the target-originated measurement falling inside the validation region.

The state in the PDAF is updated using all of the validated measurements. The update is given by

$$
\hat{x}_{k \mid k}=\hat{x}_{k \mid k-1}+K_{k} v_{k}
$$

where $\hat{x}_{k \mid k}$ is the updated state, $\hat{x}_{k \mid k-1}$ is the predicted state, $\mathrm{K}_{\mathrm{k}}$ is the Kalman gain, and $v_{\mathrm{k}}$ is the combined residual, which is given by

$$
v_{k}=\sum_{j=1}^{m_{k}} \beta_{j} v_{j k},
$$

and $v_{\mathrm{jk}}$ is the residual for the $\mathrm{j}^{\text {th }}$ validated measurement,

$$
v_{j k}=z_{j k}-H_{k} \hat{x}_{k \mid k-1} \text {. }
$$

The updated covariance is given by

$$
P_{k \mid k}=\beta_{0} P_{k \mid k-1}+\left[1-\beta_{0}\right] P_{k}^{c}+\widetilde{P}_{k}
$$

where $P_{k}^{c}=P_{k \mid k-1}+K_{k} S_{k} K_{k}^{T}$

and $\quad \tilde{P}_{k}=K_{k}\left[\sum_{j=1}^{m} \beta_{j} v_{j k} v_{j k}^{T}-v_{k} v_{k}^{T}\right] K_{k}^{T}$

\section{The Application of FDA to Target Tracking}

The conventional tracking techniques apply the "all-ornone" criterion to the tracking problem. In the target detection case, the signal detection is accomplished by comparing the target signal against a threshold. Detection is declared if the SNR exceeds a certain threshold. If the signal strength is below the threshold, the detection algorithm will classify it as a non-target.

In realistic tracking scenarios, the regions around the target detection threshold and the data association gate boundary are actually fuzzy areas where there is no clear criterion that can be applied to separate target from nontarget measurements. This fuzziness at the boundary is not an important consideration as long as the SNR is high; since the distinction between a target and nontarget can be made easily. At lower signal strength, the 
decision has to be made at the fuzzy boundaries. In this case, the bivalent logic employed by the conventional tracking techniques fails. This is one of the main reasons that no matter how sophisticated a conventional tracker is, its performance can never match the performance of an experienced human operator since the human mind is more capable of making the fuzzy decisions. The measurement to target detection, as well as target association is the result of processing the following propositions, $[13,14]$ :

1) If the SNR is high and the occurrence is close then the association degree is high.

2) If the SNR is high and the occurrence is typical then the association degree is high.

3) If the SNR is high and the occurrence is far then the association degree is moderate.

4) If the SNR is moderate and the occurrence is close then the association degree is moderate

5) If the SNR is moderate and the occurrence is typical then association degree is slightly moderate.

6) If the SNR is moderate and the occurrence is far then the association degree is low.

7) If the SNR is low and the occurrence is close then the association degree is slightly low.

8) If the SNR is low and the occurrence is typical then the association degree is moderately low.

9) If the strength is low and its occurrence is far then the association degree is very low.

10)If the SNR of all measurement signals is low then noassociation degree is high.

11)If at least one measurement SNR is moderate and its occurrence is far then the association degree is moderate.

12)If at least one measurement SNR is moderate or high and its occurrence is close or typical then noassociation degree is low.

Once the fuzzy rules have been applied, the fuzzy minmax rule of implication is used. The set membership is not a bivalent but a continuous function in real value interval $[0,1]$. The membership functions are shown in figures (3), (4), and (5).

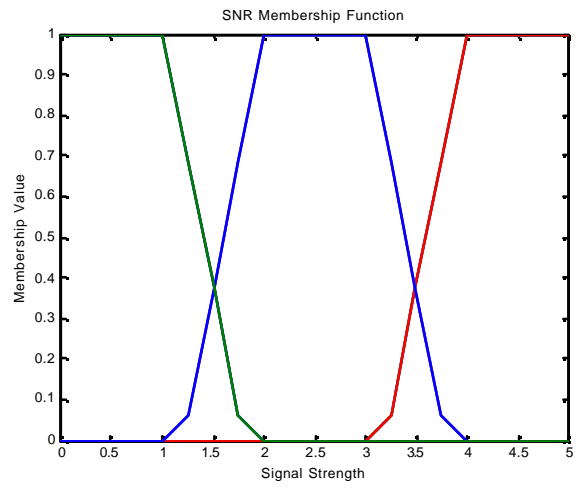

Fig.3 SNR domain fuzzy sets

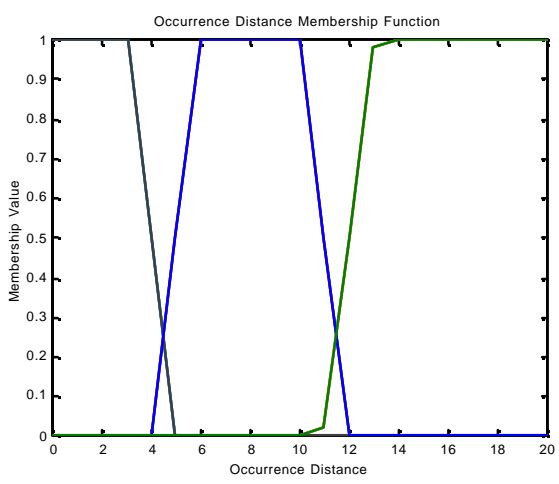

Fig. 4 Occurrence distance domain fuzzy sets

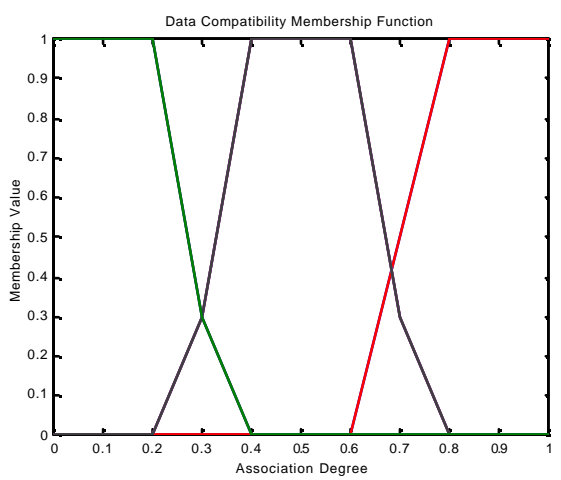

Fig. 5 Data compatibility domain fuzzy sets

\section{Simulation Results of PDA, FDA, and VDA for Target Tracking in Clutter}

In order to compare the performance of the three trackers, a true target trajectory is generated using MATLAB as shown in Figures 6 and 7. This is an example of the signal follower. The tracker tries to follow the bearing and frequency of underwater acoustic signals from single target in the presence of uniform clutter. Noise and uniform background clutter were added to the true target track to generate the corrupted target trajectory as shown in figures 8 and 9. All the results presented in this paper have been generated using MATLAB.

The simulation is performed using two different SNRs; low SNR $(-2.5 \mathrm{~dB})$ and high SNR $(5 \mathrm{~dB})$. The three trackers were tested against these two different SNRs and the results are shown in figures 10 to 21 . Figures 10 to 15 show the estimated bearing and frequency of the three trackers at high SNR. The figures show that the trackers can track the target under this condition and in the presence of clutter. Figures 16 to 21 show the estimated bearing and frequency of the three trackers at low SNR. The figures show that the VDA and the FDA trackers can track the target under this condition and in the presence of clutter while the PDA tracker fails to 
track the target when having low SNR. The root mean square (RMS) errors of the estimated bearing and frequency at low SNR are shown in figures 22 and 23 . The RMS errors are calculated by performing a 100 run Monte Carlo simulation. The RMS errors prove that the VDA and the FDA can be considered an optimal solution to the data association problem when receiving low SNR signals from the target. Also the simulations reveal that the VDA is computationally more efficient than the FDA and the PDA.

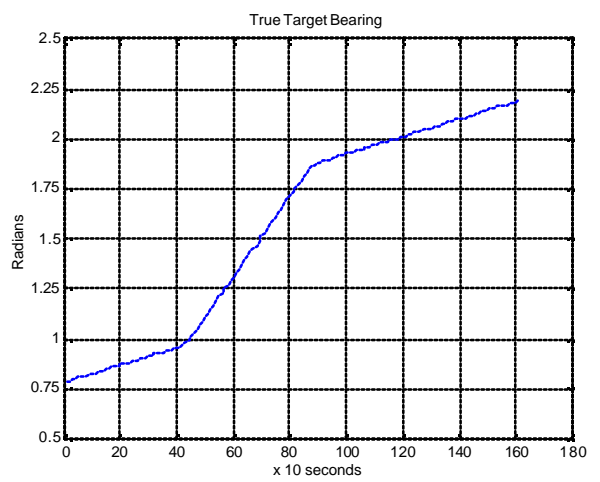

Fig. 6 True target bearing track

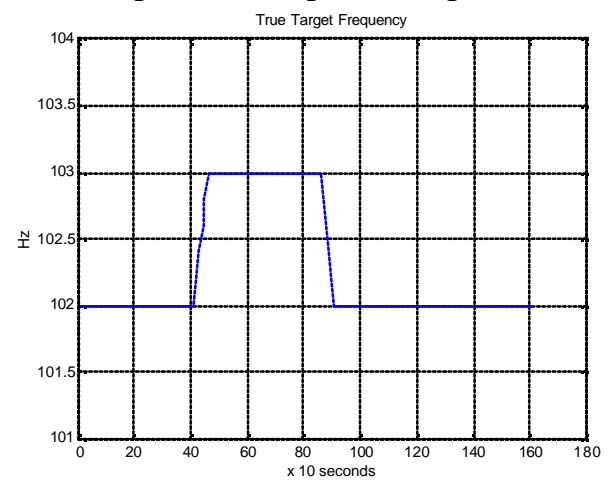

Fig. 7 True target frequency track

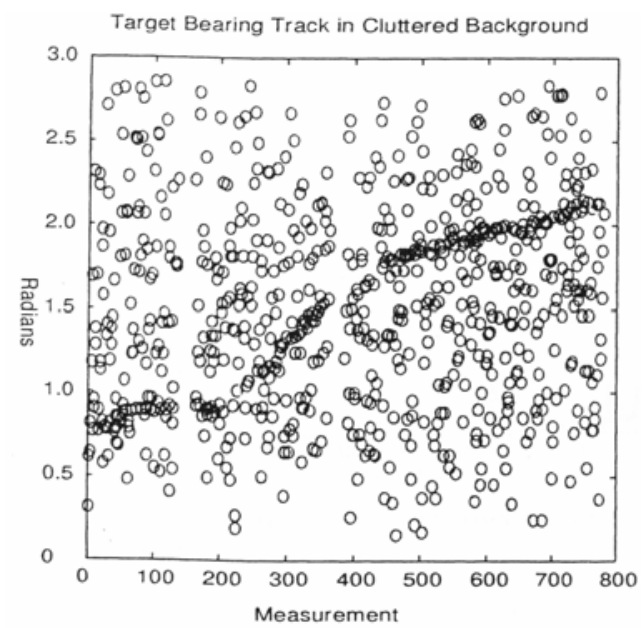

Fig. 8 Target bearing track in clutter

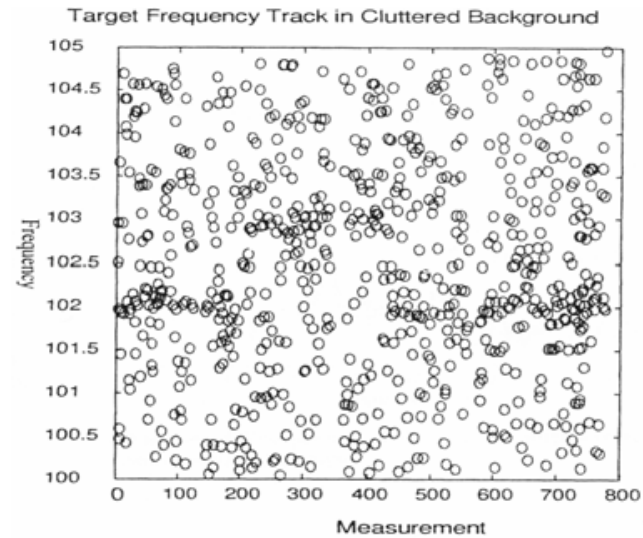

Fig. 9 Target frequency track in clutter

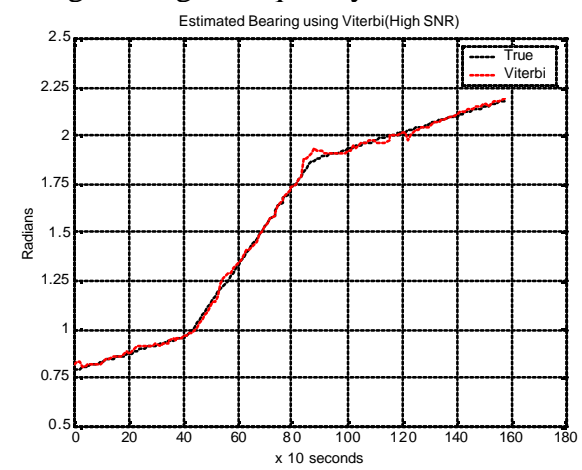

Fig. 10 Estimated bearing track using VDA (High SNR)

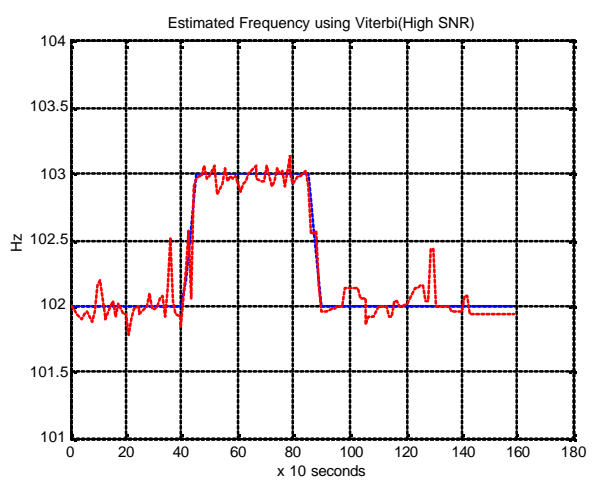

Fig. 11 Estimated frequency using VDA (High SNR)

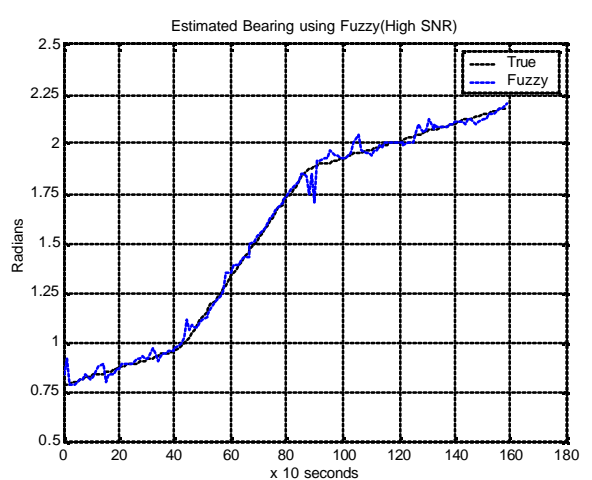

Fig. 12 Estimated bearing track using FDA (High SNR) 


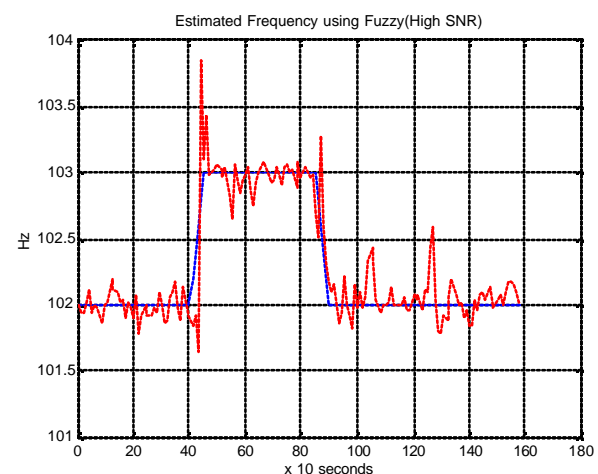

Fig. 13 Estimated frequency using FDA (High SNR)

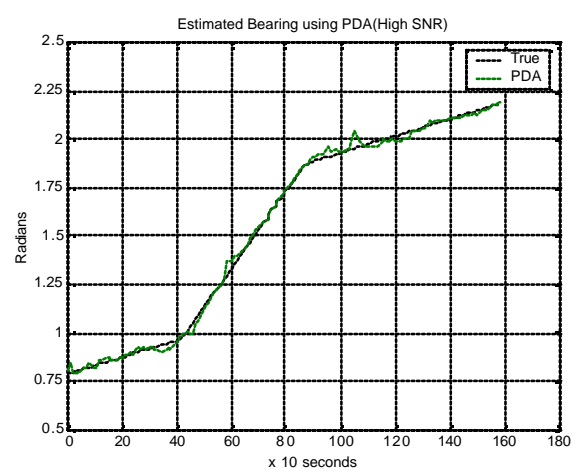

Fig. 14 Estimated bearing track using PDA (High SNR)

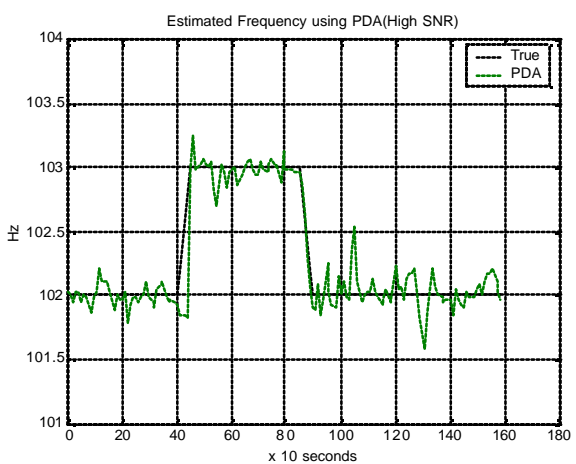

Fig. 15 Estimated frequency using PDA (High SNR)

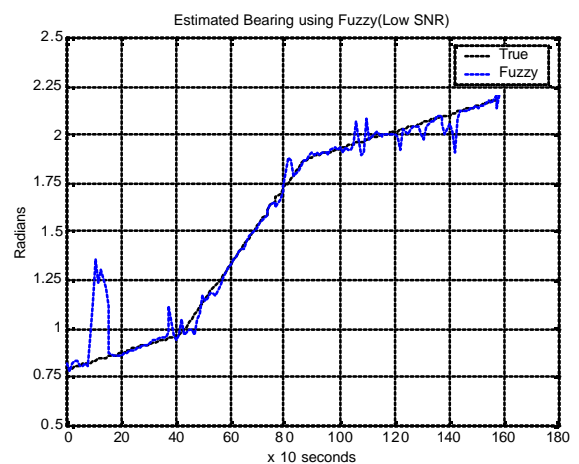

Fig. 16 Estimated bearing track using VDA (Low SNR)

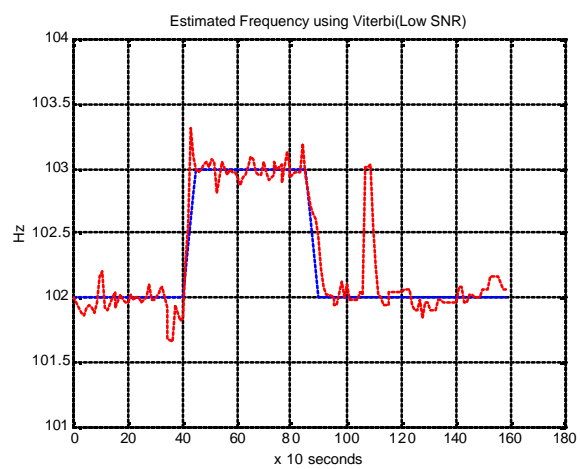

Fig. 17 Estimated frequency using VDA (Low SNR)

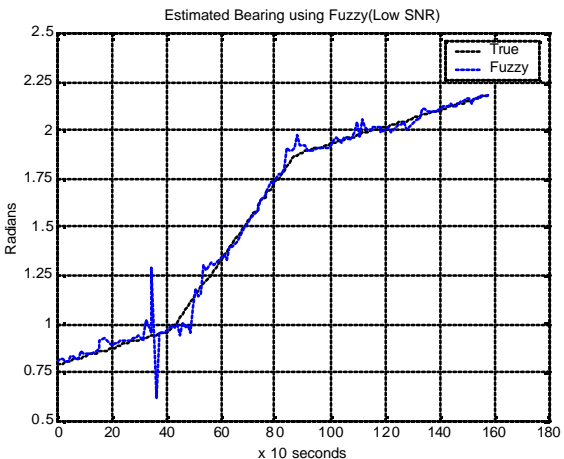

Fig. 18 Estimated bearing track using FDA (Low SNR)

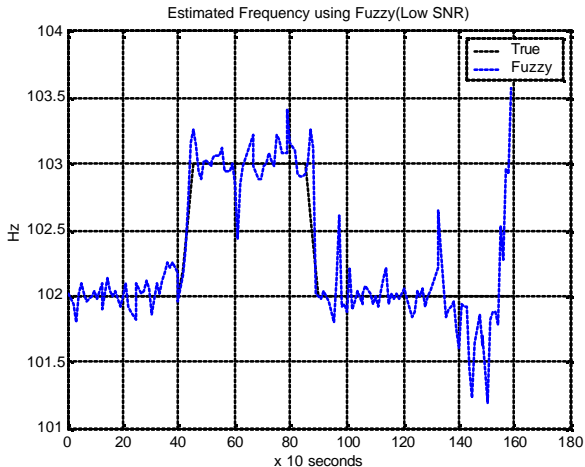

Fig. 19 Estimated frequency using FDA (Low SNR)

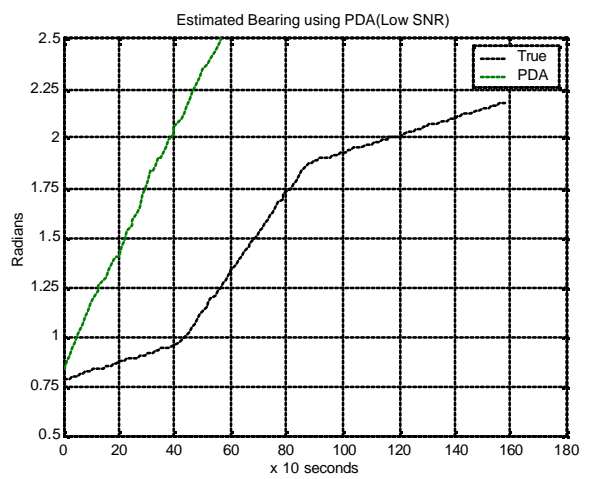

Fig. 20 Estimated bearing track using PDA (Low SNR) 


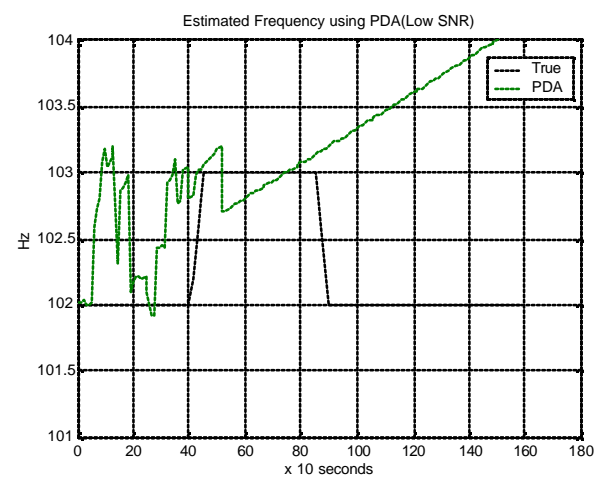

Fig. 21 Estimated frequency using PDA (Low SNR)

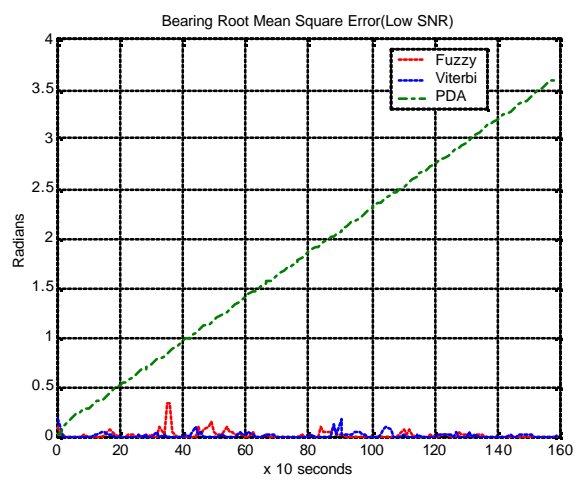

Fig. 22 Bearing RMS error (Low SNR)

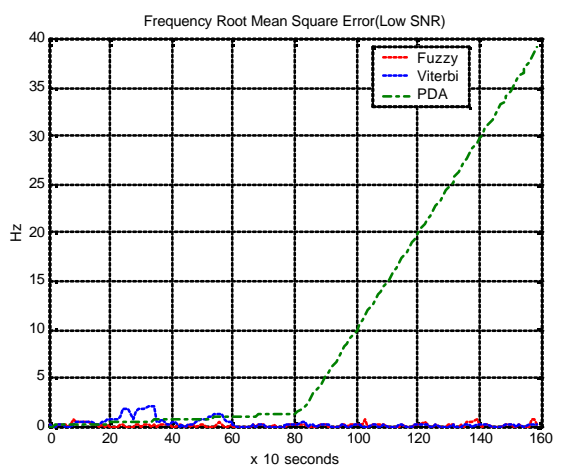

Fig. 23 Frequency RMS error (Low SNR)

\section{Conclusions and Future Work}

From the results obtained in the simulations, it can be seen that at high SNR, all the trackers (PDA, VDA, and FDA) are able to track the target in the cluttered environment. However, at low SNR, the PDA algorithm fails to track the target where both the FDA and VDA are capable of tracking the target. The VDA has the advantage of low computational cost over both the PDA and FDA.

Future work will focus on the application of both FDA and VDA in mult itarget tracking in clutter. Based on the simulation results, the VDA promises to be computationally an efficient algorithm. However, if under further tests and simulations the VDA continues to provide good tracking performance, it can be considered a comp utationally feasible alternative to the conventional trackers when tracking a considerably large number of targets

\section{References}

[1] G. Forney, "The Viterbi Algorithm", Proc. Of the IEEE, pp. 268-278, 1973.

[2] J. Omura, "On the Viterbi Algorithm", IEEE Trans. On Information Theory, pp. 177-179, 1973.

[3] H. Lou, "Implementing the Viterbi Algorithm', IEEE signal processing, pp. 42-52, Sept. 1995.

[4] T. Quach and M. Farooq, "Maximum Likelihood Track Formation with the Viterbi Algorithm", Proc. Of the $33^{\text {rd }}$ CDC, pp. 271-276, Dec. 1994.

[5] B. La Scala and G. Pulford, "A Viterbi Algorithm for Data Association", Proc. 35 ${ }^{\text {th }}$ CDC, Dec. 1996.

[6] L. Rabiner and B. Juang, "An Introduction to Hidden Markov Models", IEEE ASSP Magazine, pp. 4-15, Jan. 1986.

[7] H. Blom and Y. Bar-shalom, "The Interactive Multiple Model Algorithm for Systems with Markovian Switching Coefficients", IEEE Trans. Auto. Contr., Vol. AC-33, Aug. 1988.

[8] S. Blackman, "Multiple-Target Tracking with Radar Applications", Norwood, MA: Artech House, 1986.

[9] S. Blackman and R. Popoli, "Design and Analysis of Modern Tracking Systems", Artech House, 1999.

[10] Y. Bar-Shalom, "Multitarget-Multisensor Tracking: Advanced Applications", Artech House, 1990.

[11] Y. Bar-Shalom, "Multitarget-Multisensor Tracking: Applications and Advances, II", Artech House, '92.

[12] Y. Bar-Shalom and W. Blair, "MultitargetMultisensor Tracking: Applications and Advances Volume III", Artech House, 2000.

[13] J. Wolf, A. H. Viterbi, and G. Dixion. Finding the best set of $\mathrm{K}$ paths through a trellis with application to multi-target tracking. IEEE Trans. AES, 25(2): 287-296, 1989.

[14] T. Quach and M. Farooq, "A Fuzzy Logic-Based Target Tracking Algorithm," Proceeding of SPIE, Vol. 3350,Orlando, FL, pp. 476-487, April 1998.

[15] A. Gad, M. Farooq and S. Midwood, "Applications of Fuzzy Logic to Target Tracking in a Cluttered Environment", Proceeding of SPIE, Vol. 4380, Orlando, FL, pp. 91-104. April 2001. 